\title{
Pre-Chamber Ignition Mechanism: Experiments and Simulations on Turbulent Jet Flame Structure
}

\author{
P.M. Allison, P.M. de Oliveira, A. Giusti, and E. Mastorakos \\ *Email: pma34@cam.ac.uk \\ Department of Engineering, University of Cambridge, Cambridge CB2 1PZ, UK
}

\begin{abstract}
This work investigates the effects of premixed combustion kinematics in pre-chamber volumes on the development of emitted hot jets from the igniter. The effects of fuel type, orifice diameter, and ignition location are evaluated experimentally, with high-speed $\mathrm{OH}^{*}$ and $\mathrm{CH}^{*}$ chemiluminescence imaging, and computationally with Large-Eddy Simulations (LES). The imaging experiments allowed for simultaneous viewing of combustion processes within a quartz chamber and of the developing jet flow. Results from these experiments provided insight on the temporal evolution of the jet relative to the growth of an ignited kernel within the chamber, as well as information on the emission or lack of emission of radical species from the chamber. Computational results provided data on the temporal behavior of the pressure within the chamber and profiles of the high velocity flow through the orifice. These results, combined, have shown that dependent on the strain rate and effective orifice size, local quenching of radical species at the orifice occurs which fundamentally change whether hot products, reactive layers, or both are present in the turbulent jet emission. The dynamic structure and composition of the turbulent jet controls its relevance as an effective ignition source.
\end{abstract}

\section{Introduction}

Ignition in large-scale natural gas internal engines may be difficult to achieve with standard electrical spark systems at high pressures and lean chamber mixtures. Ignition by hot turbulent jets from a pre-chamber igniter is proving to be advantageous for these types of combustion environments. These pre-chamber systems are composed of a small volume adjoined to the combustion cylinder. Within the pre-chamber, a standard electrical spark is used to ignite a fuel-air mixture controlled by separate fuel injection in the pre-chamber or the mixture that has entered from the main chamber during the compression stroke. Dependent on the mixture and chamber geometry, a combination of reacting and quenched species are injected into the main chamber by high-speed, hot turbulent jets emerging from small orifices that connect the main and pre-chamber volumes.

This process of ignition via emerging jets from a pre-chamber is denoted as "Turbulent Jet Ignition (TJI)" in literature and has been the focus of studies on fundamental and industrial devices at lab-scale and engine-relevant conditions. Toulson et al. [1] have provided a thorough review of pre-chamber systems as prescribed to engine-based applications. Studies conducted at industrial scales are difficult to image, however several fundamental works have investigated the jet development in simpler geometries. Yamaguchi et al. [2] investigated, in a separated pre-chamber and main chamber, the ignition and burning mechanisms of the main chamber mixture by a torch jet (orifice diameters between 4 and 14mm). Recently, Biswas et al. [3, 4] studied the ignition mechanisms of $\mathrm{CH}_{4} /$ air and $\mathrm{H}_{2}$ /air mixtures and two ignition mechanisms were identified as "jet ignition" and "flame ignition", respectively corresponding to jets consisting of hot combustion products and wrinkled turbulent flames, respectively. The idea of 
a critical global Damköhler number was introduced, as a limiting parameter that separates the ignition from a no-ignition regime.

Several other experimental studies have investigated pre-chamber ignition in full scale natural gas engines and rapid compression machines (RCM) for observation at elevated pressures. A joint experimental and computation effort by Roesthlisberger and Favrat $[5,6]$ identified engine performance effects of varying pre-chamber orientation and orifice geometry within the cylinder. RCM studies [7-9] have focused on geometric effects on the ignition and flame spread within a main chamber. In particular, high speed imaging revealed the influences of orifice size and mixture composition on the jet dynamics.

The primary foci of these previous studies have been on pre-chamber mixture composition and geometry and their relation towards the ignition of some external volume or flow. In this sense, the physical application of the pre-chamber as an igniter has been of importance. However, little investigation has been placed on the fundamental nature of what occurs inside the prechamber and the evolution of the jet as a fluid dynamic feature independent of an external flammable mixture. The aim of the current work was to experimentally evaluate, in a simple geometry with complete optical access to the pre-chamber, the temporal behavior and emission of the jet as correlated with the propagation of flames imaged within the pre-chamber.

These investigations considered the effects of fuel type, mixture composition, orifice size, and ignition location in the chamber. Particular emphasis was placed on the presence of radical chemiluminescence emission from the jet to evaluate quenching of the reactive flow due to thermal losses and strain through the orifices. Large eddy simulations (LES) of the same configuration studied experimentally have also been performed to provide insight on chamber pressure and velocity through the orifice and how these may relate to quenching in the jet. The structural nature of the jet composition as a combination of fresh mixture, reactive layers, and hot products is essential to the performance of pre-chambers as ignition sources. These combined experimental and computational efforts provide further fundamental understanding of the dynamic and regimental behavior of pre-chamber ignition processes.

\section{Experimental and Numerical Methods}

The pre-chamber apparatus used is shown in Figure 1. It consists of a quartz cylinder with an inner diameter of $32 \mathrm{~mm}$ and a length of $32 \mathrm{~mm}$, constrained at both ends with flat, $1.5 \mathrm{~mm}$ thick steel plates. This volume forms the "pre-chamber". The quartz cylinder allows for complete optical access to the pre-chamber and the ability to image the evolution of the confined flame growth. The baseplate has a series of sharp-edged holes which are used as the exit orifice. These orifices allow for the selection of chamber exits with diameters of $1.5 \mathrm{~mm}, 3 \mathrm{~mm}$ or $6 \mathrm{~mm}$. The other plate has an opening fit with a solenoid valve, which is used for filling the pre-chamber with the mixture to be ignited. In the images, an apparent $4 \mathrm{~mm}$ gap between the bottom of the chamber and beginning of the jet is seen. This viewing blockage is due to the combined thicknesses of the end plate and sealing gasket. The premixed equivalence ratio of the mixture inside is varied between 0.9 and 1.2 for methane-air and ethylene-air mixtures. The fluid into which the jet emerges is air at ambient conditions. Table 1 provides the operating conditions and geometric configurations over which experiments were conducted.

Ignition is provided by a spark created by a focused $532 \mathrm{~nm}$ laser beam from a Continuum Surelite II Nd:YAG laser at $50 \mathrm{~mJ} /$ pulse. Laser ignition allows for precise control of the spark location and for this fundamental study removes any physical obstructions to the kernel growth due to ignition hardware. A similar strategy was employed for pre-chamber studies by Joshi et 
al [10]. The laser is focused at two different centerline locations within the chamber; $16 \mathrm{~mm}$ from the orifice exit (center of the chamber) and $30 \mathrm{~mm}$ from the orifice exit at the mixture inlet (top of the chamber). For ignition at the top of the chamber, the beam is focused by a $100 \mathrm{~mm}$ focal length through the orifice and impinges on the pre-chamber top endplate. The laser beam is delivered from a $50 \mathrm{~mm}$ focal length lens for the chamber-centered ignition location through the side of the quartz cylinder. This creates the ignition basis kernel with a toroidal shape characteristic of laser ignited mixtures $[11,12]$. Ignition at the top of the chamber does not create a toroidal kernel. It appears that the spark interaction with the wall creates a hemispherical ignition kernel which propagates toward the orifice.

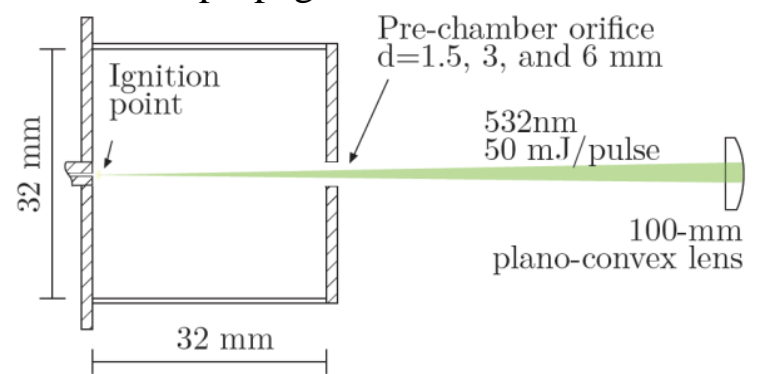

Figure 1. Pre-chamber jet igniter schematic and ignition method

Table 1. Experimental test conditions and evidence of radical presence in the jet.

\begin{tabular}{|c|c|c|c|c|c|}
\hline \multirow{2}{*}{ Fuel } & $\begin{array}{c}\text { Orifice Diameter } \\
{[\mathbf{m m}]}\end{array}$ & $\begin{array}{c}\text { Ignition } \\
\text { Location }\end{array}$ & $\begin{array}{c}\text { Equivalence } \\
\text { Ratio }\end{array}$ & Jet OH* & Jet CH* \\
\hline \multirow{5}{*}{ Methane } & 1.5 & Top & $0.9,1.0,1.2$ & $\checkmark$ (low signal) & $\mathbf{x}$ \\
\cline { 2 - 6 } & 3 & Top & $0.9,1.0,1.2$ & $\checkmark$ & $\mathbf{x}$ \\
\cline { 2 - 6 } & 6 & Top & $0.9,1.0,1.2$ & $\checkmark$ & $\checkmark$ \\
\cline { 2 - 6 } & 3 & Center & 1.0 & $\checkmark$ & $\mathbf{x}$ \\
\hline \multirow{4}{*}{ Ethylene } & 6 & Center & 1.0 & $\checkmark$ & $\checkmark$ \\
\cline { 2 - 6 } & 1.5 & Top & $0.9,1.0,1.2$ & $\checkmark$ & $\checkmark$ \\
\cline { 2 - 6 } & 3 & Top & $0.9,1.0,1.2$ & $\checkmark$ & $\checkmark$ \\
\hline
\end{tabular}

High speed chemiluminescence imaging of the $\mathrm{OH}^{*}$ and $\mathrm{CH}^{*}$ radicals were performed independently at $8 \mathrm{kHz}$ with a Photron SA.1 and LaVision HS-IRO high speed intensifier. The imaging system was operated with an exposure time of $120 \mu$ s and an intensifier gain of 60 . The imaging region captured both the optically accessible pre-chamber and the exiting jet into the ambient. Due to the large difference in signal contrast between the pre-chamber and jet chemiluminescence, an OD 0.2 neutral density filter was used to mitigate chemiluminescence signal from the chamber. The imaging field-of-view was $75 \mathrm{~mm}$ wide by $120 \mathrm{~mm}$ tall with a resolution of $115 \mu \mathrm{m} / \mathrm{pixel}$. Table 1 lists whether, for the given sensitivity of the imaging system, if $\mathrm{OH}^{*}$ or $\mathrm{CH}^{*}$ were seen in the jet. A typical turbulent jet structure may include a combination of flame and hot products emerging into fresh mixture which has been ejected ahead of the growing flame kernel. $\mathrm{OH}^{*}$ signal is being used to track heat release and the presence of products, whereas $\mathrm{CH}^{*}$ marks flame reaction zone [13]. The absence of $\mathrm{CH}^{*}$, in particular, from the jet suggests quenching of reactive layers in the orifice. In this paper, a few of these results are shown in the form of extracted stills and quantitatively analyzed for correlations between the chamber kinematics and jet evolution, with the aim to reveal the location and shape of the reaction zone at various stages of the flame development.

The openFOAM package (version 2.3) has been used for modelling the methane flames. Cases with different diameter of the orifice and different location of the ignition have been considered in this work. The sub-grid scale stress tensor was modelled with the constant Smagorinsky 
model. The combustion model proposed by Weller et al. [14] was used. This model is based on the solution of the filtered regress variable together with a transport equation for the sub-grid flame wrinkling. Although the premixed flame model used here may not fully account for flame quenching due to heat loss or high stretch, and hence may not reveal fully the details of the flow and flame at the nozzle, the simulations provide some basic flow patterns during the flame expansion process. Furthermore, some useful indications regarding the global behavior of the pre-chamber (e.g. the time evolution of the pressure) for the different configurations can be extracted, complementing the experimental measurements in the understanding of the main characteristics of the jet development. The computational domain includes the ignition chamber and extends downstream of the orifice for about 8d. The domain was discretized with a hexahedral mesh of about 9.3 million cells. Adiabatic no-slip conditions were applied to solid walls; wave transmissive condition was used for the pressure at the far field boundary (downstream of the nozzle) in order to avoid reflection of pressure fluctuations. Second-order schemes were used for spatial discretization whereas the time derivative was discretized with a first-order implicit scheme. Adjustable time step was used during the simulation to maintain the maximum Courant number lower than 0.4 in the entire domain. The resulting time step was in the range $10^{-9}-10^{-7} \mathrm{~s}$ for the time period simulated in this work. The simulation was initialised with a quiescent stoichiometric mixture of methane at atmospheric pressure. The computation of the reaction term in the regress variable equation requires the evaluation of the laminar flame speed which was computed using the correlation by Gulder [15]. Note that this correlation may need improvement at engine-relevant conditions [16]. A spark (modelled as a sink term in the regress variable equation) of duration $50 \mu \mathrm{s}$ and diameter $5 \mathrm{~mm}$ was imposed at the beginning of the simulation to initialize the flame. No tuning of this simplified ignition treatment was attempted to match the experiment, since the focus was on the subsequent flame development process. Therefore, the simulations do not reproduce exactly the experimental ignition process, but it is expected that the simulation of evolving flame can be qualitatively comparable with the experiment.

\section{Results and Discussion}

Figures 2 and 3 depict ethylene ignition sequences and jet formations for the $3 \mathrm{~mm}$ and $6 \mathrm{~mm}$ orifices, respectively. Figure 5 shows similar sequences from the LES effort of the temperature field of the propagating methane kernels due to top and center ignition. These particular image series show the initial growth of the flame kernel from a laser spark occurring at the center of the chamber. The kernel is not perfectly spherical due to the toroidal plasma shape formed during the laser ignition. This is caused by the interaction between the rarefaction wave and the hot gas kernel produced by the spark, and is followed by the development of a "third lobe", which then grows in the direction of the laser beam [12]. In addition, due to the presence of the orifice, a pressure gradient is created towards the chamber exit. For cases where kernel formation occurs at the top of the chamber due to the laser impingement, the kernel is more spherical in shape. Both ignition locations generate a laminar flame that propagates through the quiescent mixture in the chamber.

As the kernel initially grows and the pressure rises, reactant mixture from the chamber is ejected. The kernel continues to grow, propagating towards the orifice and corners of the chamber. Once the flame has reached the orifice exit, a jet, as indicated by both $\mathrm{OH}^{*}$ and $\mathrm{CH}^{*}$, emerges from the pre-chamber. For the ethylene, the jet is seen almost within $1 \mathrm{~ms}$ after the initial laser spark. Over time, the length of the jet increases with increasing consumption of the mixture within the chamber. However, as will be discussed in the forthcoming section, the evolution of the jet is highly dependent on the pressure in the chamber and velocity field at the orifice. These flow field characteristics are determined by the initial equivalence ratio, fuel type, 
orifice diameter, and spark location. The jet length as indicated by the $\mathrm{OH}^{*}$ signal typically reaches its maximum length at the peak fuel consumption of the chamber.

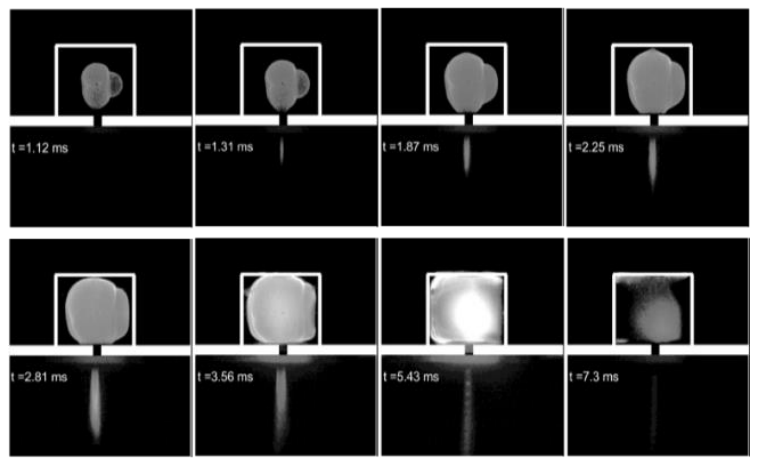

Figure 2. $\mathrm{OH}^{*}$ ignition sequence for the $3 \mathrm{~mm}$ orifice, ethylene, $\phi=1$ [17].

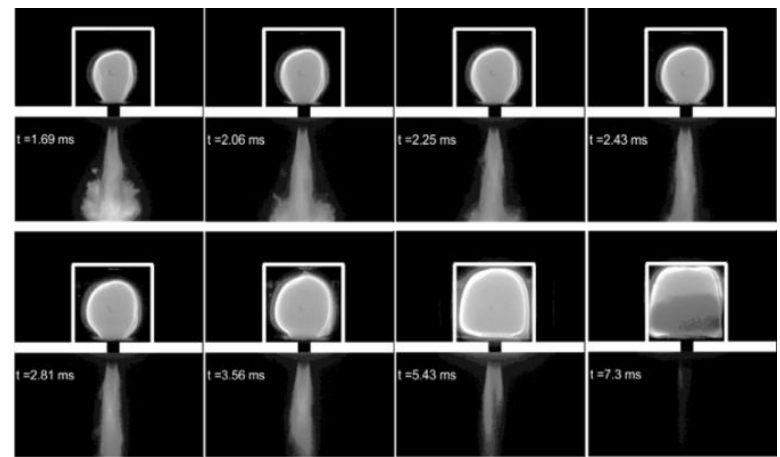

Figure 3. $\mathrm{OH}^{*}$ ignition sequence for the $6 \mathrm{~mm}$ orifice, ethylene, $\phi=1$ [17].

However, it should also be noted that quenching occurs as the flame propagates towards the walls and corner, limiting the consumption of reactant mixtures. Thus depending on the initial conditions, $100 \%$ of the reactant mixture may never be consumed, such as those seen in Figures 2 and 3. There are certain cases, though not pictured, where after the flame fully contacts the walls of the chamber, portions of the flame quench on the quartz; small reacting segments may continue to propagate into the corners.

Figure 4 depicts the ignition sequence of methane in the $3 \mathrm{~mm}$ orifice chamber. Visualizations of both $\mathrm{OH}^{*}$ and $\mathrm{CH}^{*}$ are presented to illustrate the quenching effects that begin once the orifice diameter approaches the limits of the flame thickness and quenching distance. As given in Table $1, \mathrm{OH}^{*}$ is seen for all orifice diameters. However, $\mathrm{CH}^{*}$, which is indicative of the excited radical produced in the reactive layers of the flame front, may be quenched and not seen in the imaging. This is true of the $3 \mathrm{~mm}$ case for methane and the $1.5 \mathrm{~mm}$ case for both fuels. The lower row of Figure 4 shows this particular process where $\mathrm{CH}^{*}$ is clearly seen in the chamber flame, however no radical emission is captured outside of the chamber. The presence of flame reaction zones in the jet is controlled by the degree of flame quenching through the orifice which is a process is controlled by the quenching distance, as determined by the flame speed, pressure, and density [18], in relation to the orifice diameter. For low turbulence, such as these quiescent chambers, theory shows that the quenching distance scales proportionally with the temperature and inversely proportional with the flame speed and pressure. In the experiments presented so far, stoichiometric ethylene and methane was used in the pre-chamber. The flame speed of $\mathrm{C}_{2} \mathrm{H}_{4}$ at atmospheric conditions is about $0.8 \mathrm{~m} / \mathrm{s}$, while that of $\mathrm{CH}_{4}$ is about $0.4 \mathrm{~m} / \mathrm{s}$ [19]. The flame thickness is proportionally thicker for methane. The quenching distance for methane at ambient conditions is about 2-3mm [20], with a flame thickness about $1 \mathrm{~mm}$ [21], whereas ethylene's quenching distance is approximately $1 \mathrm{~mm}$. The smaller selected orifices have diameters close 
to the nominal quenching distances for these fuels. With the added LES information on slightly elevated pressures but high temperatures in Fig. 6 and 7, the nominal quenching distance at reacting conditions in the orifice is increased. At these conditions the quenching distance is larger than the orifice and this is verified by quenching of $\mathrm{CH}^{*}$ in the small diameters for methane and ethylene.

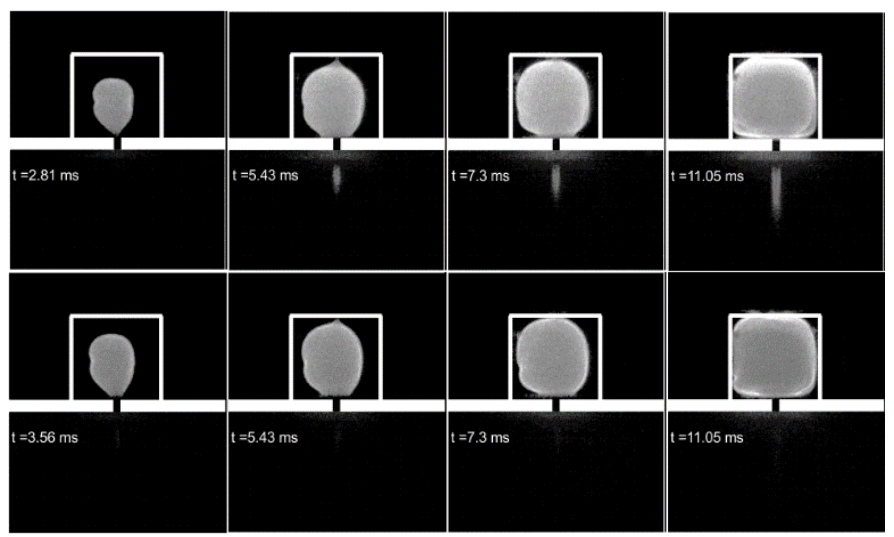

Figure 4. $\mathrm{OH}^{*}$ ignition sequence for the $3 \mathrm{~mm}$ orifice (top row), methane, $\phi=1$. $\mathrm{CH}^{*}$ ignition sequence for the $3 \mathrm{~mm}$ orifice (bottom row), methane, $\phi=1$.

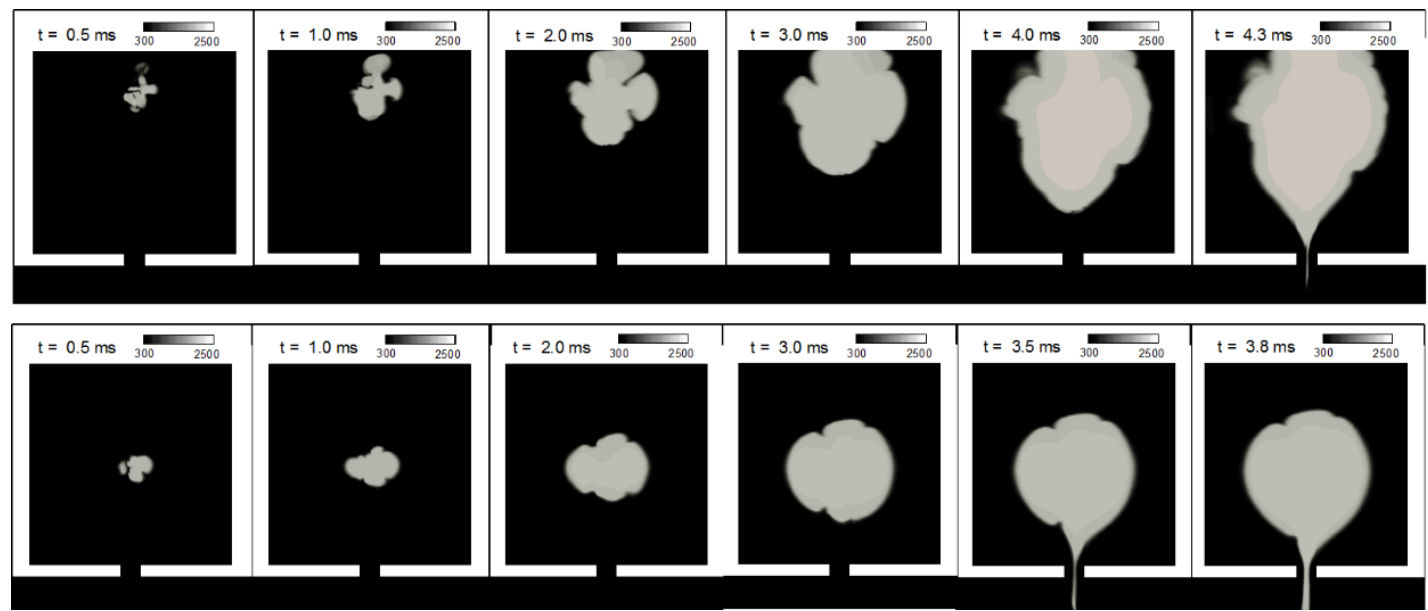

Figure 5. Temperature profiles of kernel formation from LES for top (top row) and center (bottom row) ignition sequences for the $3 \mathrm{~mm}$ orifice, methane, $\phi=1$.

The growth of the kernel inside the pre-chamber predicted by LES is shown in Figure 5 for cases characterized by different ignition locations. In agreement with the experiment (Figure 4 and 8), hot products reach the orifice first in the case of ignition at the center of the pre-chamber and in the case with larger orifice diameter. The arrival times of the centrally-ignited methane kernels at the orifice are also similar $(\sim 3.2 \mathrm{~ms})$ between the experiment and LES. It is important to point out that the fluid flowing out of the orifice consists of both fresh reactants and combustion products, with the latter located in the core of the jet and the former in the region adjacent to the orifice wall. Therefore the reaction zone (at the interface of these two layers) is of a cylindrical shape, with diameter smaller than the orifice diameter.

The pressures predicted by the computational simulations indicate large differences in varying the orifice diameters as seen in Figure 6. A large pressure drop across the orifice, together with high temperature (low density) combustion products, results in very high velocity flows through the orifices. Velocity profiles for cases with different diameters of the orifice are shown in Fig. 
7(b,d). High velocities are usually located at the center of the jet, where low-density flow is located, with low velocity flow of fresh reactants close to the walls. At the earliest instant for the $3 \mathrm{~mm}$ case, a high velocity is measured, but no temperature rise, which suggests the initial flow of fresh reactants out of the chamber ahead of the reacting kernel. The simulations also show that the sharp corners of the orifice create small-scale regions of recirculation at the orifice wall. Analysis has shown that these regions decrease the effective area of the orifice up to $20 \%$ and further increase the centerline velocity. Flame quenching can occur due to both thermal quenching by heat transfer to the walls and radical quenching. Both these mechanisms can also be enhanced in the presence of high strain rates (dU/dr), which are achieved in the cases observed. This feature enhances the quenching experienced in the orifice. The simulations show that for this geometry, the flow though the orifice approach high Mach numbers. At $5.43 \mathrm{~ms}$, in Figure 4, a pattern of high and low intensities is visible in the $\mathrm{OH}^{*}$ jet. Similar structure is seen in the imaging work of Biswas et al [4]. This suggests that perhaps in the experiments, when evaluated at times longer than the LES simulation when the Mach number may be higher, the non-adiabatic orifice may begin to choke. This fluctuation in the local mass flow rate may lead to the quenching pattern observed.

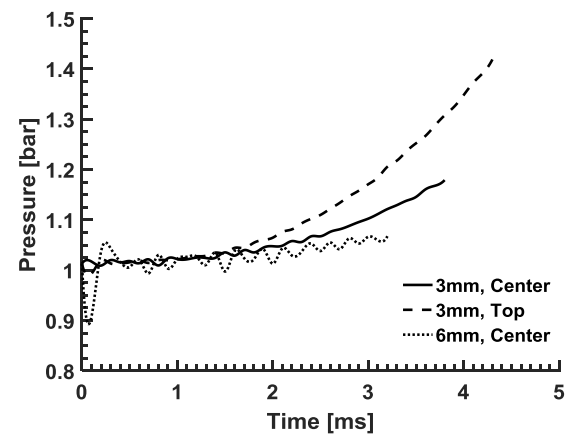

Figure 6. Initial transient pre-chamber pressure predicted by LES for varying orifice diameter and ignition location, methane, $\phi=1$.
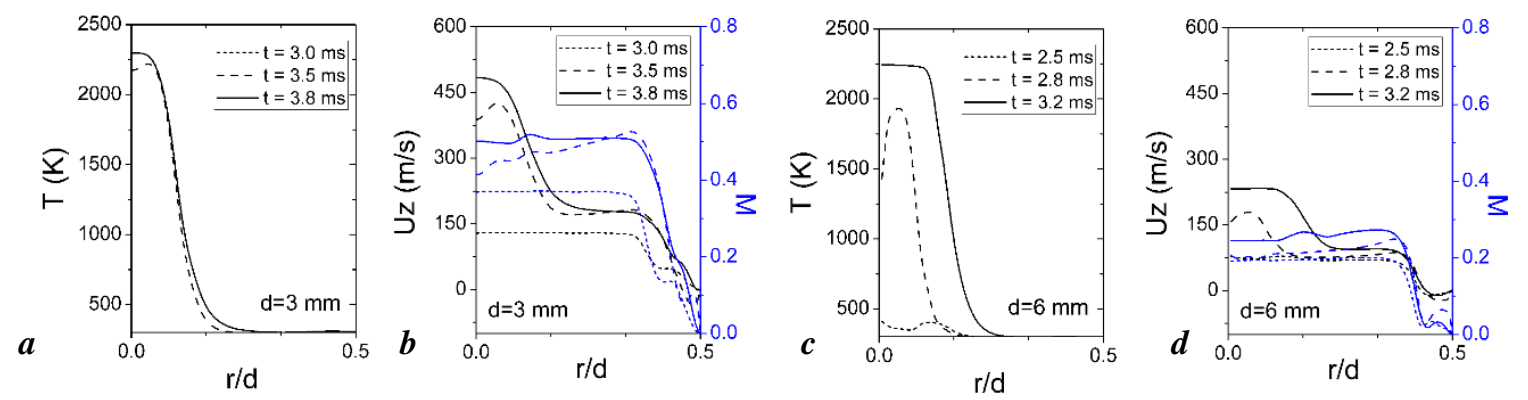

Figure 7. Temperature and velocity profiles across the orifice radius for various instances after ignition for $3 \mathrm{~mm}$ orifice $(a, b)$ and for $6 \mathrm{~mm}$ orifice $(c, d)$, methane, $\phi=1$.

Figure 8 shows the effect of the spark location of the formation of the emerging jet, from the experiment. The ethylene jets appear earlier in time than the methane jets because of the difference in flame speed and higher chamber pressures due to higher adiabatic flame temperatures. This is also clearly seen in the LES images of Figure 5. When the spark is created at the center of the chamber, the propagating flame reaches the orifice earlier than when the flame propagates initially from the top of the chamber. As well, the duration of the jet is longer for center ignition. This may be due to the difference in consumption of mixture in the chamber such that less pre-chamber fluid has already been ejected before the jet flame appears. 


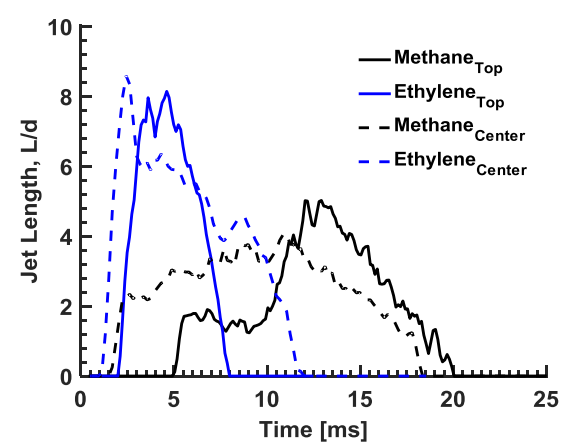

Figure 8. Jet lengths calculated from $\mathrm{OH}^{*}$ chemiluminescence depicting the effect of spark location. Lengths are normalized by the $3 \mathrm{~mm}$ diameter, for methane and ethylene, $\phi=1$.

Figure 9 depicts the effect of equivalence ratio on the evolution of the jet in time. It appears that for ethylene mixtures (Fig 9, left), there is no effect on the initial start time of the jet, as compared to the methane mixtures (Fig 9, right). For ethylene, the strongest effect is on the maximum jet length which occurs for the richest case. For the methane mixtures, the richest case shows a long delay in jet emission compared to the leaner cases. However, the richest case still produces the longest jet. The increase jet length in the richer cases may be explained by the presence of significant flammable mixture present in the ambient ahead of the hot product jet. External ignition of this ambient fluid in the richer case may lead to the appearance of extended jet signals.

With visualization in the chamber, the growth of the flame can be temporally tracked. Figure 10 illustrates the growth of the flame as it consumes the mixture, and the mean $\mathrm{OH}^{*}$ signal in the chamber over time. The consumed volume of reactant mixture is calculated by estimating the margins of the fully-enclosed flame surface through binarization of the line-of-sight $\mathrm{OH}^{*}$ signal. However, it should be noted that due to the initial ejection of fluid from the chamber due to the pressure rise, the total flammable mass within the chamber varies temporally and dependent on the operating conditions. The radial margins of the kernel along the axial direction of the chamber demarcate the width of a disk of 1 pixel $(115 \mu \mathrm{m})$ height. These disks are presumed to be individually symmetric about their own axis, which is a decent assumption given the near-spherical growth of top-side ignition. The volume of each of these revolved disks is integrated along the axial direction to give the total volume encompassed by the boundaries of the $\mathrm{OH}^{*}$ signal. This "consumed" volume is then compared to the total volume. It must be stated that this method provides a reasonable estimate of the consumed volume up until the flame kernel arrives at the walls and begins to quench, at which point the kernel may no longer form an enclosed burnt volume. The limit at which this occurs is indicated by the transition of the consumption plot to a constant maximum value. Values less than $100 \%$ indicate that the flame kernel expanded to wall and began to quench prior to complete consumption of mixture in the corner. In some cases, the consumed volume may asymptotically approach $100 \%$ if small flame fragments propagate into the corners. However, this complete consumption occurs inconsistently, and for this discussion these asymptotic effects are neglected.

For ethylene mixtures, the consumption rate is similar for each tested equivalence ratio. As well, the mean signal in the chamber is largest for the stoichiometric cases of ethylene and methane. For the methane mixtures, complete propagation across the chamber volume by the single kernel is not achieved. This again suggests that the flame has not propagated into the corners of the chamber. This is particularly true for the Phi $=1.2$ case, which also displays a roughly $6 \mathrm{~ms}$ delay in behavior. The delay is concurrently observed by the delay in jet 
appearance in Figure 9. There is a large difference in the timescales over which these ignition sequences occur. For ethylene mixtures, the entire chamber is ignited and totally void of $\mathrm{OH}^{*}$ signal within $10 \mathrm{~ms}$. For the methane mixtures, this same process take place over $20-40 \mathrm{~ms}$. In addition, the point of maximum consumption is also correlated with the time of maximum mean $\mathrm{OH}^{*}$ signal in the chamber. This is consistent with the method of volume estimation, such that when the flame has expanded to its largest singular volume, the total signal will also be maximized.
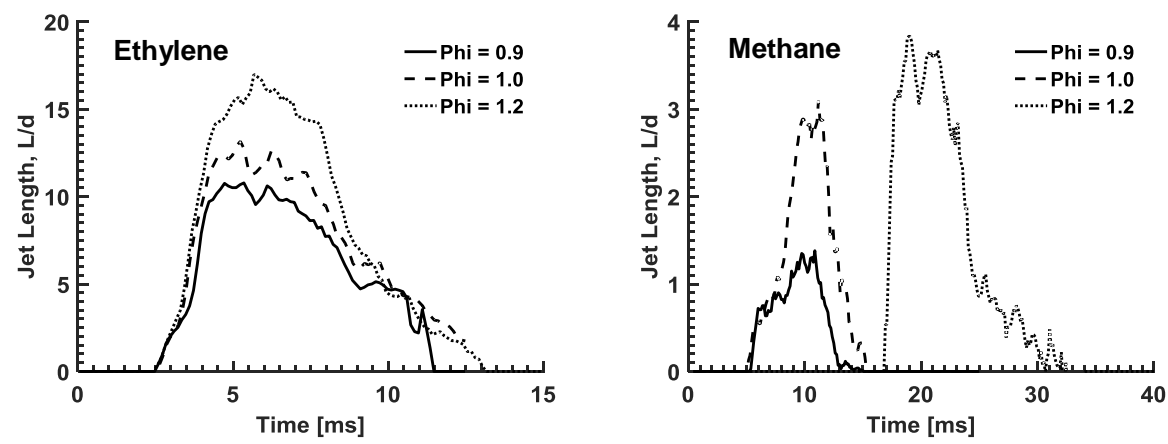

Figure 9. Jet lengths calculated from $\mathrm{OH}^{*}$ chemiluminescence depicting the effect of equivalence ratio for top ignition. Lengths are normalized by the $3 \mathrm{~mm}$ diameter, for ethylene (left) and methane (right).
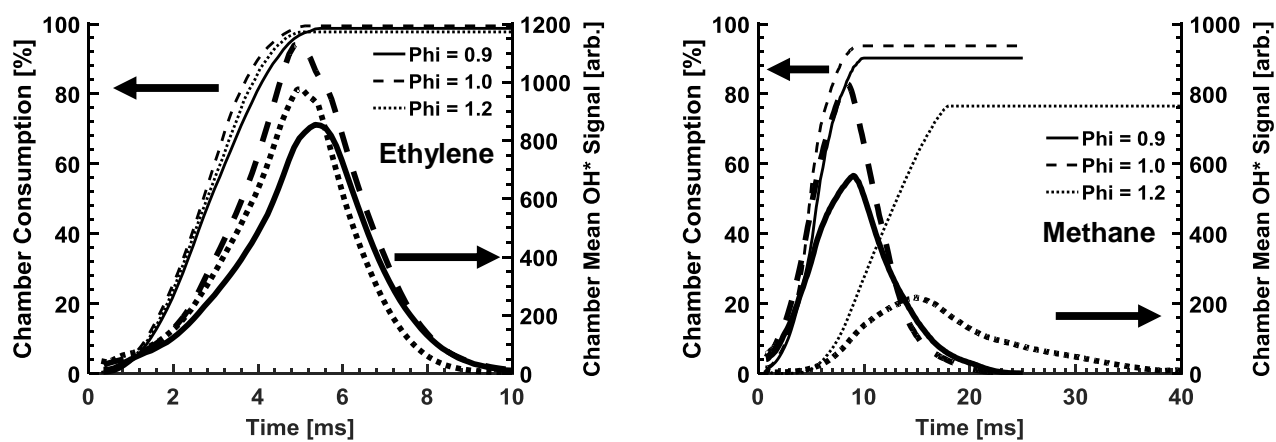

Figure 10. Volume of mixture consumed within the pre-chamber as a percent of the total chamber volume, (left axes, thin lines) and mean $\mathrm{OH}^{*}$ signal intensity in chamber (right axes, bold lines) depicting the effect of equivalence ratio for top ignition in the $3 \mathrm{~mm}$ orifice, for ethylene (left) and methane (right).

Similar observations of jet length and chamber consumption can be observed for varying orifice diameter, as seen in Figures 11 and 12. Figure 11 (left) shows the difference in jet evolution for methane and ethylene. Jet emission is observed at earlier time for the ethylene flames due to the faster arrival of the flame kernel at the orifice. The differences between the orifice sizes can be supported by the differences in chamber pressures and exit velocities predicted in the LES. It can also be seen that for the larger diameters, the jet length in methane cases initially rises and then plateaus for some milliseconds before rising to their maximum values respectively. The reason behind this behavior is not immediately clear, nor is it apparent from the consumption plots in Figure 12.

Figure 11 (right) shows lengths for the ethylene cases that exhibit both $\mathrm{OH}^{*}$ and $\mathrm{CH}^{*}$ presence in the turbulent jets. The presence of both radicals suggests that hot products and flame reaction zone have successfully propagated out of the chamber without quenching in the orifice. However, there are clear differences in the spatial and temporal structure of each jet marker. 
For both diameters, the appearance of $\mathrm{CH}^{*}$ occurs after a $1 \mathrm{~ms}$ delay from first observations of the $\mathrm{OH}^{*}$ jet. In addition, the $\mathrm{CH}^{*}$ decays away earlier than the $\mathrm{OH}^{*}$ jet. The physical length of the $\mathrm{CH}^{*}$ jet is consistently shorter than the $\mathrm{OH}^{*}$ jet. Structurally this strongly suggests a reacting cylindrical core jet surrounded by hot products, as conceptually seen in Figure 13. For cases where quenching occurs, this reacting core is absent. As the reacting jet exits the orifice, it is preceded by hot products from the chamber, as well as fresh mixture that was ejected as the pressure initially began to rise.

The consumption of chamber mixture and mean $\mathrm{OH}^{*}$ signals for each orifice size are given in Figure 12. Similar to the equivalence ratio variation, there are large differences between the timescales and signal intensities observed. For ethylene cases, the peak consumption, occurs roughly at the same time ( $\sim 5 \mathrm{~ms}$ after ignition), however there is a wider variation of consumption rates for the methane cases, such that the $3 \mathrm{~mm}$ orifice chamber is consumed fastest and most completely. The total ethylene ignition sequences are longer with decreasing orifice size, whereas the opposite trend is observed in methane. Comparison with the LES consumption rate (Fig. 12, red) for the $3 \mathrm{~mm}$ methane case based on Figure 5 shows good agreement from top-side ignition to the moment the kernel reaches the orifice.
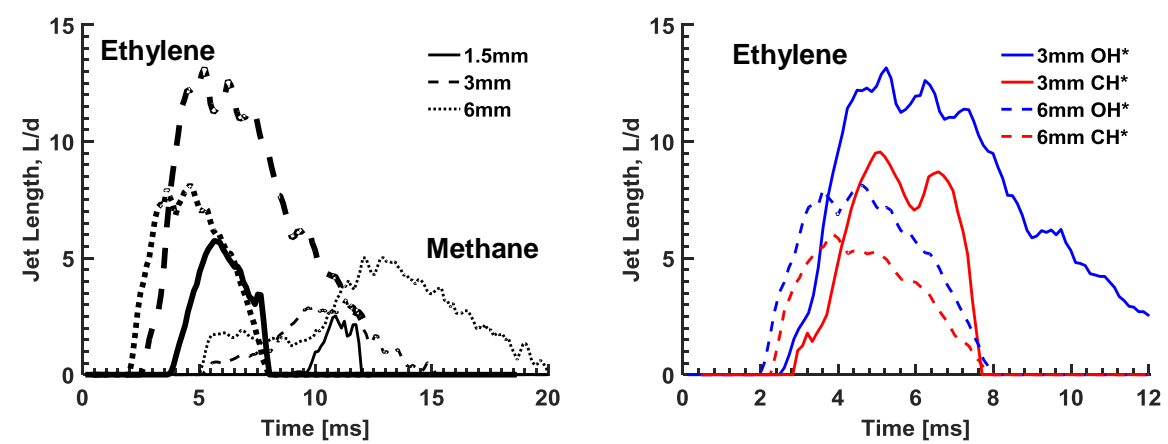

Figure 11. (Left) Jet lengths calculated from $\mathrm{OH}^{*}$ chemiluminescence depicting the effect of orifice diameter for top ignition, $\phi=1$. Ethylene in bold lines. Methane in thinner lines.

(Right) Comparison of jet lengths calculated from $\mathrm{OH}^{*}$ and $\mathrm{CH}^{*}$ in ethylene, $\phi=1$ mixture for $3 \mathrm{~mm}$ and $6 \mathrm{~mm}$ orifice sizes.
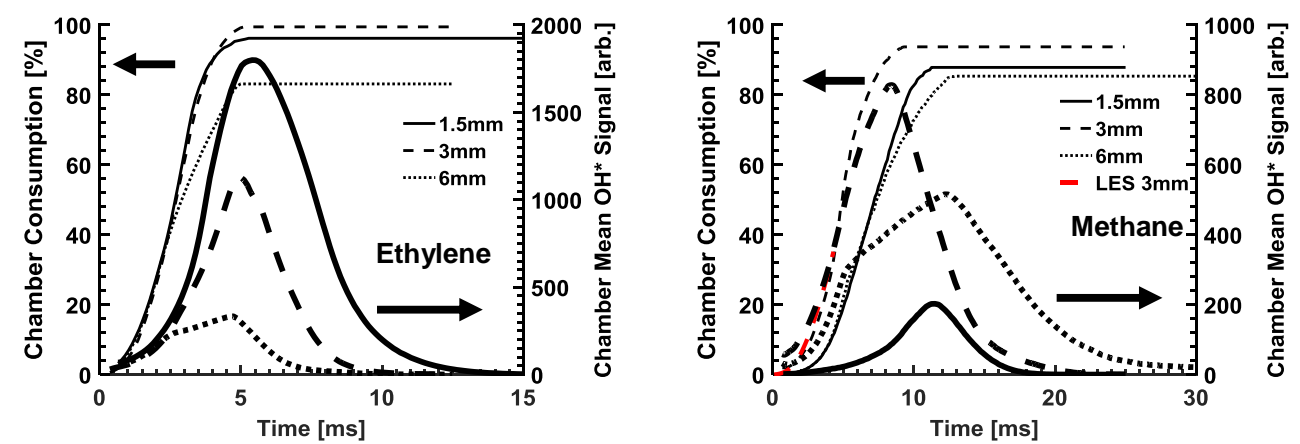

Figure 12. Volume of mixture consumed within the pre-chamber as a percent of the total chamber volume, (left axes, thin lines) and mean $\mathrm{OH}^{*}$ signal intensity in chamber (right axes, bold lines) depicting the effect of orifice diameter for top ignition, $\phi=1$, for ethylene (left) and methane (right). LES volume consumption comparison (right, red) for the $3 \mathrm{~mm}$ methane case.

Figure 14 depicts the correlation of the $\mathrm{OH}^{*}$ jet lengths matched temporally with the consumption of mixture within the chamber. For both fuels, it can be seen that the larger diameter orifices emit jet signal at smaller kernel sizes. Turbulent jets are not observed in the 
small diameter chambers until almost all $(+70 \%)$ of the chamber has been burnt. However, the respective start points of methane and ethylene are similar per diameter. The largest difference between the fuels is seen in the jet length plateaus that occur shortly after first appearance of the jets in methane cases. These plots also suggest that for top-side ignition, the jet length is maximized at the point of maximum consumption for all cases. As well, for all cases, the jet lengths continue to decay in size once the majority of the chamber has been consumed. This is consistent with the idea that no further hot products are available to be convected out of the chamber. Furthermore, the decrease in heat release within the chamber will be coupled with a decrease in pressure and temperature, causing a reduction in the jet velocity.
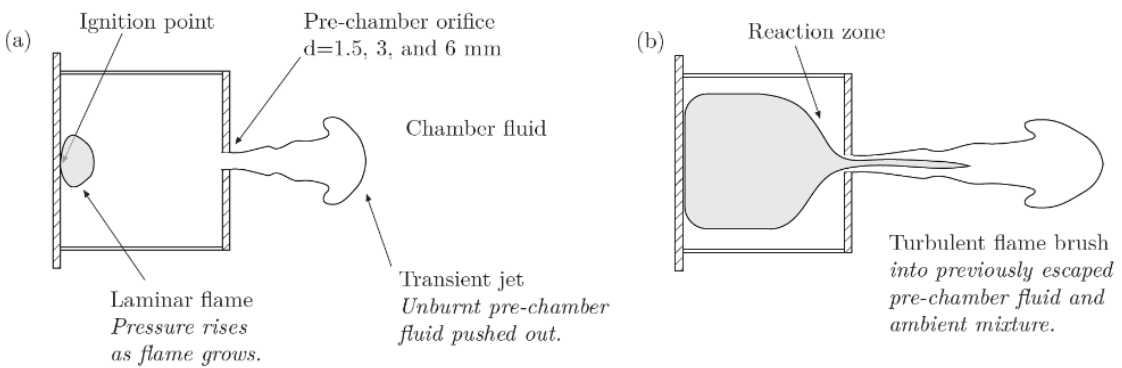

Figure 13. Kernel ignition/growth in which hot products and reactive layers are emitted [17].
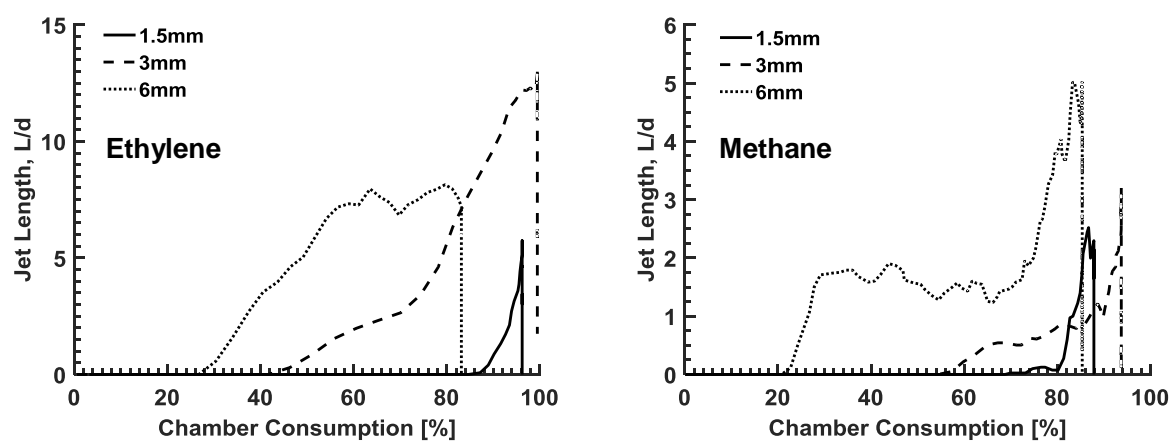

Figure 14. Measured jet lengths temporally correlated with the volume of mixture consumed within the pre-chamber as a percent of the total chamber volume, depicting the effect of orifice diameter for top ignition, $\phi=1$, for ethylene (left) and methane (right).

\section{Conclusions}

In this work, fuel type, orifice geometry, and ignition location have shown strong consequences regarding three main factors affecting pre-chamber igniters: 1) Kernel development and propagation internal to the chamber, 2) Turbulent jet composition and temporal evolution, and 3) Quenching of radical species representative of reaction zone penetration from the orifice. Jet penetration length, jet duration, and reaction zone presence are strongly linked to the performance of these igniter devices. The development and propagation of a flame which consumes the mixture internal to the chamber is dependent initially on the mixture equivalence ratio and ignition location. Higher flame speeds, such as in ethylene, affect the consumption rate of the chamber, which has been shown to be correlated with the timing of the initial jet appearance. Decreasing orifice size and ignition location are factors which also strongly control the jet emission timing and the pressure rise in the chamber. This pressure rise along with the change in density/temperature of the reacting flow leads to high velocities from the orifice and longer jets lengths. The compositional structure of the jets are a combination of reactive layers and/or hot products as seen in imaging of $\mathrm{CH}^{*}$ and $\mathrm{OH}^{*}$ radicals. However, the relative scales of fuel quenching distances and small orifice diameters, when coupled with high strain rates, may result in complete quenching of these emitted reaction zones. LES results also demonstrate 
that recirculation patterns in the orifice can further reduce the effective diameter of the orifice which enhances any quenching tendencies.

\section{Acknowledgements}

We wish to thank Prof. K. Boulouchos and Drs. C. Frouzakis and Y.M. Wright of ETH Zurich, for their hospitality during a sabbatical stay of one of us (EM) and for useful discussions on this problem. PMO would like to thank the financial support of the Brazilian Space Agency and Brazil's National Council for Scientific and Technological Development

\section{References}

[1] Toulson, E., Schock, A.J., Attard, W.P., "A review of pre-chamber initiated jet ignition combustion systems", SAE Paper 2010-01-2263 (2010).

[2] Yamaguchi S., Ohiwa, N. and Hasegawa, T., "Ignition and Burning Process in a Divided Chamber Bomb," Combustion and Flame 59: 177-187 (1985).

[3] Biswas S., Tanvir S., Wang H., and Qiao L., "On ignition mechanisms of premixed CH4/air and H2/air using a hot turbulent jet generated by pre-chamber combustion", Applied Thermal Engineering 106: 925937 (2016).

[4] Biswas, S. and Qiao, L., "Prechamber hot jet ignition of ultra-lean $\mathrm{H}_{2}$ /air mixtures: Effect of supersonic jets and combustion instability," SAE Int. J. Engines 9(3) (2016).

[5] Roethlisberger, R.P. and Favrat, D., "Investigation of the prechamber geometrical configuration of a natural gas spark ignition engine for cogeneration: part 1. Numerical simulation," International Journal of Thermal Sciences 42, 223-237 (2003).

[6] Roethlisberger, R.P. and Favrat, D., "Investigation of the prechamber geometrical configuration of a natural gas spark ignition engine for cogeneration: part 1. Experimentation," International Journal of Thermal Sciences 42, 239-253 (2003).

[7] Gentz, G., Thelen, B., Gholamisheeri, M., Litke, P, Brown, A., Hoke, J., and Toulson, E., “A study of the influence of orifice diameter on a turbulent jet ignition system through combustion visualization and performance characterization in a rapid compression machine," Applied Thermal Eng 81:399-411 (2015).

[8] Gholamisheeri, M., Thelen, B.C., Gentz, G.R., Wichman, I S., and Toulson E., "Rapid compression machine study of a premixed, variable inlet density and flow rate, confined turbulent jet," Combustion and Flame 169:321-332 (2016).

[9] Schlatter, S., Schneider, B., Wright, Y.M., and Boulouchos, K., "Comparative Study of Ignition Systems for Lean Burn Gas Engines in an Optically Accessible Rapid Compression Expansion Machine", SAE technical paper no. 2013-24-0112, doi:10.4271/2013-24-0112.

[10] Joshi, S., Loccisano, F., and Montgomery, D.T., "On comparative performance testing of prechamber and open chamber laser ignition,” J. Eng. Gas Turbines Power 133 (2011).

[11] Spiglanin, T.A., Mcilroy, A., Fournier, E., Cohen, R.B., and Syage, J.A., "Time-resolved imaging of flame kernels: laser spark ignition of H2/O2/Ar mixtures", Combustion and Flame 102: 310-328 (1995).

[12] Bradley, D., Sheppard, C.G.W., Suardjaja, I.M., and Woolley, R., "Fundamentals of high-energy spark initiation with lasers", Combustion and Flame 138: 55-77 (2004).

[13] Guethe, F., Guyot, D., Singla, G., Noiray, N., and Schuermans, B., "Chemiluminescence as diagnostic tool in the development of gas turbines," Appl Phys B 107:619-636 (2012).

[14] Weller, H.G., Tabor, G., Gosman, A.D., Fureby, C., "Application of a flame-wrinkling LES combustion model to a turbulent mixing layer", Symposium (International) on Combustion, 27:889-907 (1998).

[15] Gulder, O.L., "Correlations of laminar combustion data for alternative SI engine fuels", SAE Technical Paper 841000 (1984).

[16] Xu, G., Hanauer, C., Wright, Y.M., and Boulouchos, K., "CFD-Simulation of Ignition and Combustion in Lean Burn Gas Engines”, SAE Technical paper No. 2016-01-0800, doi:10.4271/2016-01-0800.

[17] Allison, P.M., de Oliveira, P.M., Giusti, A., Mastorakos, E., Benekos, S., Wright, Y.M., Frouzakis, C., and Boulouchos, B., "Fundamental aspects of jet ignition for natural gas engines," under review for SAE ICE 2017.

[18] Ballal, B. and Lefebvre, A.H., "Ignition and flame quenching in flowing gaseous mixtures," Proc. R. Soc. Lond. A. 375:163-181 (1977).

[19] Law, C.K., "Combustion Physics", Cambridge University Press, 2006.

[20] Sotton, J., Boust, B., Labuda, S.A., and Bellenoue, M., "Head-on quenching of transient laminar flame: heat flux and quenching distance measurements," Combust. Sci. and Tech., 177: 1305-1322 (2005).

[21] Jarosinski, J., "A survey of recent studies on flame extinction,” Prog. Energy Combust. Sci., 12: 81-116 (1986). 